\title{
Cerebral near infrared spectroscopy oximetry in extremely preterm infants: phase II randomised clinical trial
}

Simon Hyttel-Sorensen research fellow ${ }^{1}$, Adelina Pellicer associate professor ${ }^{2}$, Thomas Alderliesten research fellow ${ }^{3}$, Topun Austin consultant neonatologist ${ }^{4}$, Frank van Bel professor of Neonatology ${ }^{3}$, Manon Benders consultant neonatologist ${ }^{35}$, Olivier Claris professor ${ }^{6}$, Eugene Dempsey professor ${ }^{7}$, Axel R Franz associate professor ${ }^{8}$, Monica Fumagalli consultant neonatologist ${ }^{9}$, Christian Gluud head of department ${ }^{10}$, Berit Grevstad trial manager ${ }^{11}$, Cornelia Hagmann consultant neonatologist ${ }^{12}$, Petra Lemmers consultant neonatologist ${ }^{3}$, Wim van Oeveren managing director ${ }^{13}$, Gerhard Pichler associate professor ${ }^{14}$, Anne Mette Plomgaard research fellow ${ }^{1}$, Joan Riera biomedical engineer ${ }^{215}$, Laura Sanchez consultant neonatologist ${ }^{2}$, Per Winkel senior researcher ${ }^{10}$, Martin Wolf professor ${ }^{16}$, Gorm Greisen professor ${ }^{1}$

\footnotetext{
${ }^{1}$ Department of Neonatology, Rigshospitalet, Copenhagen University Hospital, Blegdamsvej 9, DK-2100 Copenhagen, Denmark; ${ }^{2}$ Department of Neonatology, La Paz University Hospital, Madrid, Spain; ${ }^{3}$ University Medical Center Utrecht, Wilhelmina Children's Hospital, Utrecht, Netherlands; ${ }^{4}$ Rosie Hospital, Cambridge University Hospitals NHS Foundation Trust, Cambridge, UK; ${ }^{5}$ Centre of the Developing Brain, King's College London, London, UK; ${ }^{6}$ Department of Neonatology, Hopital Femme Mere Enfants, Bron, France; ${ }^{7}$ Department of Paediatrics and Child Health, University College Cork, Cork, Republic of Ireland; ${ }^{8}$ Department of Neonatology, University Children's Hospital Tübingen, Tübingen, Germany; ${ }^{9} \mathrm{NICU}$, Fondazione IRCCS Ca' Granda Ospedale Maggiore Policlinico, Milan, Italy; ${ }^{10}$ Copenhagen Trial Unit, Centre for Clinical Intervention Research, Rigshospitalet, Copenhagen University Hospital, Copenhagen, Denmark; ${ }^{11}$ Centre for Clinical Intervention Research, Rigshospitalet, Copenhagen University Hospital, Copenhagen, Denmark; ${ }^{12}$ Division of Neonatology, University of Zurich, Zurich, Switzerland; ${ }^{13}$ Haemoscan, Groningen, Netherlands; ${ }^{14}$ Department of Pediatrics, Medical University of Graz, Graz, Austria; ${ }^{15}$ Center for Biomedical Technology, Technical University of Madrid, Madrid, Spain; ${ }^{16}$ Biomedical Optics Research Laboratory, Division of Neonatology, University Hospital Zurich, Zurich, Switzerland
}

\begin{abstract}
Objective To determine if it is possible to stabilise the cerebral oxygenation of extremely preterm infants monitored by cerebral near infrared spectroscopy (NIRS) oximetry.

Design Phase II randomised, single blinded, parallel clinical trial.

Setting Eight tertiary neonatal intensive care units in eight European countries.

Participants 166 extremely preterm infants born before 28 weeks of gestation: 86 were randomised to cerebral NIRS monitoring and 80 to blinded NIRS monitoring. The only exclusion criterion was a decision not to provide life support.

Interventions Monitoring of cerebral oxygenation using NIRS in combination with a dedicated treatment guideline during the first 72
\end{abstract}

hours of life (experimental) compared with blinded NIRS oxygenation monitoring with standard care (control).

Main outcome measures The primary outcome measure was the time spent outside the target range of $55-85 \%$ for cerebral oxygenation multiplied by the mean absolute deviation, expressed in \%hours (burden of hypoxia and hyperoxia). One hour with an oxygenation of $50 \%$ gives $5 \%$ hours of hypoxia. Secondary outcomes were all cause mortality at term equivalent age and a brain injury score assessed by cerebral ultrasonography.

Randomisation Allocation sequence $1: 1$ with block sizes 4 and 6 in random order concealed for the investigators. The allocation was stratified for gestational age ( $<26$ weeks or $\geq 26$ weeks).

Blinding Cerebral oxygenation measurements were blinded in the control group. All outcome assessors were blinded to group allocation. 
Results The 86 infants randomised to the NIRS group had a median burden of hypoxia and hyperoxia of $36.1 \%$ hours (interquartile range 9.2-79.5\%hours) compared with 81.3 (38.5-181.3) \%hours in the control group, a reduction of $58 \%$ (95\% confidence interval $35 \%$ to $73 \%$, $\mathrm{P}<0.001)$. In the experimental group the median burden of hypoxia was 16.6 (interquartile range 5.4-68.1) \%hours, compared with 53.6 (17.4-171.3) \%hours in the control group $(\mathrm{P}=0.0012)$. The median burden of hyperoxia was similar between the groups: 1.2 (interquartile range 0.3-9.6) \%hours in the experimental group compared with 1.1 (0.1-23.4) \%hours in the control group ( $\mathrm{P}=0.98)$. We found no statistically significant differences between the two groups at term corrected age. No severe adverse reactions were associated with the device.

Conclusions Cerebral oxygenation was stabilised in extremely preterm infants using a dedicated treatment guideline in combination with cerebral NIRS monitoring.

Trial registration ClinicalTrial.gov NCT01590316.

\section{Introduction}

About 25000 infants are born extremely preterm every year in Europe. Mortality of such infants is up to $20 \%$, and about $25 \%$ of survivors will have cerebral palsy or cognitive impairment. ${ }^{1-3}$ The first hours and days of a preterm infant's life carry the greatest risks of cerebral hypoxia and hyperoxia, because the transition of the respiratory and circulatory systems just after birth is associated with haemodynamic instability. ${ }^{4}$ Low systemic blood flow and fluctuations in systemic blood flow during these first days of life are associated with poor neurodevelopmental outcomes. ${ }^{56}$ To reduce the risk of impaired neurodevelopment, effective preventive measures are needed.

Near infrared spectroscopy (NIRS) oximetry enables non-invasive estimation of regional tissue haemoglobin oxygen saturation $\left(\mathrm{rStO}_{2}\right)$ because the ratio of the concentrations of oxygenated haemoglobin to total haemoglobin in the penetrated tissue are in the range of $0 \%$ to $100 \% .^{7}$ The NIRS sensor is placed over the organ of interest; the $\mathrm{rStO}_{2}$ reflects primarily the venous saturation of the blood in a volume of tissue to a depth of about $2 \mathrm{~cm} .{ }^{8}$ NIRS offers a direct, continuous measure of the oxygen balance of the organ. $\mathrm{rStO}_{2}$ of the brain has been shown to correlate with cerebral blood flow in adults and in infants born at term. ${ }^{9}{ }^{10}$ The thin skull of preterm infants makes cerebral NIRS measurements well suited for this population.

Data on the association of cerebral $\mathrm{rStO}_{2}$ and long term outcomes are limited in preterm infants. One study found low cerebral $\mathrm{rStO}_{2}$ associated with lower developmental score at 2 years of age in infants born very preterm. ${ }^{11}$ Two other studies found that a low cerebral $\mathrm{rStO}_{2}$ on the first day of life was associated with higher grade germinal matrix-intraventricular haemorrhage. ${ }^{12} 13$ In a piglet model it was shown that a low cerebral $\mathrm{rStO}_{2}$ must be maintained some time before manifest brain damage occurs. ${ }^{14}{ }^{15}$ Furthermore, preclinical data suggest that hyperoxia damages the immature brain. ${ }^{16}{ }^{17}$ These results indicate that both hypoxia and hyperoxia of the brain should be avoided, if possible.

The Safeguarding the Brains of our smallest Children (www. safeboosc.eu) project aims at testing the benefits and harms of cerebral NIRS in the first days of life in extremely preterm infants. Cerebral $\mathrm{rStO}_{2}$ monitoring and a dedicated treatment guideline ${ }^{18}$ are the cornerstones of a brain oriented protection strategy, with the goal of reducing periods of deleterious imbalance between oxygen delivery and consumption. The pilot showed that clinical implementation of this combined intervention in a single centre was feasible. ${ }^{19}$ We designed a phase II trial to test the hypothesis that the burden of hypoxia and hyperoxia could be reduced by the combination of cerebral
NIRS monitoring and a dedicated treatment guideline, and to demonstrate the feasibility of conducting a multicentre randomised clinical trial with a complex intervention in infants across Europe.$^{20}$ The ultimate goal would be to increase the survival of preterm infants without severe neurodevelopmental impairment.

\section{Methods}

This is a phase II randomised, blinded, feasibility trial of a medical device. The protocol is published elsewhere ${ }^{20}$ and is available in full at www.safeboosc.eu. It was approved by each hospital's local research ethics committee, and, where required (Austria, Denmark, France, and Switzerland) by the competent authority responsible for medical devices. An independent data and safety monitoring committee reviewed the interim safety results. The study was monitored locally according to the International Conference on Harmonisation guideline for good clinical practice. $^{21}$

\section{Participants}

Participants were recruited from the departments of neonatology by the attending neonatologists or the local research staff in Hopital Femme Mere Enfants, Lyon, France; Rigshospitalet, Copenhagen, Denmark; La Paz University Hospital, Madrid, Spain; Cork University Maternity Hospital, Cork, Ireland; Wilhelmina Children's Hospital, Utrecht, The Netherlands; Medical University of Graz, Graz, Austria; Fondazione IRCCS Ca' Granda Ospedale Maggiore Policlinico, Milan, Italy; and Rosie Hospital, Cambridge University Hospitals, United Kingdom.

Inclusion criteria were infants born more than 12 weeks before term (gestational age $<27$ weeks and six days) with a decision to provide full life support and the possibility to start cerebral NIRS monitoring within three hours after birth. Written informed consent from the parents was mandatory before inclusion and randomisation.

\section{Cerebral NIRS monitoring}

Based on data recorded with the cerebral oximeter INVOS 5100c using the adult SomaSensor (SAFB-SM) (Covidien, Boulder, $\mathrm{CO}$ ) on more than 400 preterm infants at the Wilhelmina Children's Hospital in Utrecht, the normal range of $\mathrm{rStO}_{2}$ in stable very preterm infants has been defined to be from $55 \%$ to $85 \%$ during the first 72 hours of life (Petra Lemmers and Frank van Bel, Utrecht, unpublished data). This range is considered safe and the boundary thresholds for possible interventions as listed in our treatment guidelines. ${ }^{18}$

Some NIRS devices and sensors have systematic differences..$^{22-24}$ We tested the suitability of devices by comparing absolute values, repeatability, and sensitivity to changes in oxygenation on the adult forearm. ${ }^{25}{ }^{26}$ The predefined criteria were absolute values and a dynamic range within $5 \%$ points of the INVOS oximeter and absolute repeatability better than $6 \%$ (within participant standard deviation) for acceptability of devices. Eligible devices were the INVOS 5100C with adult SomaSensor, NIRO 300, and NIRO 200NX with small probe holder (Hamamatsu Phototonics, Hamamatsu City, Japan), and the NONIN EQUANOX 7600 with adult sensor, model 8004CA (Nonin Medical, Plymouth, MN).

\section{Interventions}

We randomised the infants to either the experimental group with cerebral NIRS monitoring with visible reading of $\mathrm{rStO}_{2}$ or 
the control group with cerebral NIRS monitoring with blinded $\mathrm{rStO}_{2}$. Monitoring was started within three hours of birth and ended at 72 hours of life. In both groups the $\mathrm{rStO}_{2}$ was recorded every five or six seconds, depending on the device. In the experimental group an $\mathrm{rStO}_{2}$ alarm warned when a burden of $0.2 \%$ hours $(12 \% \mathrm{~min}$ ) had accumulated during the past 10 minutes. ${ }^{19}$ A dedicated treatment guideline (see supplementary file) was available for the clinical staff, listing possible interventions to normalise an out of range $\mathrm{rStO}_{2}$. The interventions were focused on respiratory and circulatory support. It was emphasised that all other variables, such as blood pressure, arterial saturation, and partial pressure of carbon dioxide should be kept within the target ranges in use in each unit. The treatment guideline is described in detail elsewhere. ${ }^{18}$

\section{Randomisation and masking}

The web based randomisation was set up and handled by the Copenhagen Trial Unit according to a computer generated allocation sequence of 1:1 with block sizes 4 and 6 in random order concealed for the investigators. The allocation was stratified for gestational age ( $<26$ weeks or $\geq 26$ weeks). Singleton infants were randomised individually and twins were allocated to the same treatment group. If both twins could not be included owing to lack of equipment, we included the infant born last. Owing to a lack of NIRS devices in each unit, higher order of multiple births could not be randomised.

The NIRS devices were either locked in a box, or we used a device specific research mode $\mathrm{rStO}_{2}$ blinding. All data were sent real time to a laptop. Software developed specifically for the study could either show or conceal the current $\mathrm{rStO}_{2}$ depending on group allocation. Attending clinical staff manually entered the details of repositioning of the sensor, as well as physiological variables, such as blood pressure, mean airway pressure, and blood gas variables $(\mathrm{pH}$, partial pressure of carbon dioxide, and partial pressure of oxygen. In addition, the treatment interventions during the intervention period such as treatment of a patent ductus arteriosus and number of blood transfusions were recorded in the case record forms in both groups.

\section{Outcomes, adherence, and adverse events}

The prespecified primary outcome was the time spent outside the target range of $55-85 \%$ multiplied by the mean absolute deviation during the first 72 hours after birth (burden of hypoxia and hyperoxia), expressed in percentage hours (\%hours) (fig $1 \Downarrow$ ). One hour with an oxygenation of $50 \%$ gives $5 \%$ hours of hypoxia. This figure could range from 0 to $3960 \%$ hours (55\% $\times 72$ hours) per participant. The burden was computed blinded to allocation group from the raw $\mathrm{rStO}_{2}$ data and extrapolated to 72 hours. There was no manual removal of artefacts in the $\mathrm{rStO}_{2}$ data as there are no reliable ways for these to be identified.

The secondary outcomes were all cause mortality at term equivalent age and a brain injury score determined by ultrasonography, as assigned by central, blinded reading of still images of 13 prespecified slices (coronal and sagittal) at ages $1,4,7,14$, and 35 days and at term equivalent age. This brain injury score is based on the occurrence and severity of intracranial haemorrhages, white matter abnormalities, ventricular dilation, and cerebral atrophy and is described in detail in the protocol (www.safeboosc.eu). The secondary outcome amplitude integrated electroencephalography has not yet been analysed and will be reported separately. Exploratory outcomes were serious and non-serious adverse reactions, burden of hypoxia, burden of hyperoxia, bronchopulmonary dysplasia (defined as the need for supplementary oxygen at 36 weeks of gestation), necrotising enterocolitis modified Bell's stage 2 or $3,{ }^{27}$ and retinopathy of prematurity stage 3 or worse. ${ }^{28}$

\section{Statistical analyses Sample size estimation}

The sample size estimation was based on unpublished data on 23 extremely preterm infants with a mean burden of 76.0 (SD 83.2) \%hours. We log transformed the data to achieve a normal distribution with a mean of 1.64 (SD 0.50). The sample size estimation was based on the assumptions to detect a $50 \%$ reduction or more in burden of hypoxia and hyperoxia (mean difference of 0.3 after $\log$ transformation), a type I error ( $\alpha)$ of $5 \%$, a power of $95 \%$, a twin proportion of $30 \%$, and an intraclass correlation coefficient of 0.33 . $^{29}$ This required a total sample size of 166 infants, approximately 83 per intervention group.

\section{Analyses}

All analyses were based on the intention to treat principle and conducted blinded, with two sided tests at the 0.05 level of significance. A mixed model for continuous outcome variables with random intercepts corresponding to "birth clusters" and ordinary logistic regression for binary (and ordinal) outcome variables was planned per protocol. We adjusted the analyses for the protocol specified variables (gestational age category and trial centre). The former model was not significantly different from a model without random intercept $(\mathrm{P}=1.00)$ when used for the adjusted analysis of the primary outcome. The intraclass correlation of "birth clusters" was 0.027 . All variables showed only minor differences between the models. Therefore we used the reduced model without "birth clusters" in all further analyses. Multiple imputation was not necessary because of few missing values. We adjusted the $\mathrm{P}$ values for the secondary outcomes with Hommel's procedure for multiplicity. Calculations with different hypothetical $P$ values for the planned third secondary outcome (amplitude integrated electroencephalography) were done, aiming for the most conservative adjustment - that is, the highest adjusted $\mathrm{P}$ values.

\section{Results}

Between 1 June 2012 and 31 December 2013 we screened 370 infants for eligibility, and 166 were randomised (fig $2 \Downarrow$ ). In four instances only one twin could be included. Two infants in the control group did not have any NIRS data recorded; one had consent withdrawn and there were technical problems for the other. All other data were collected per protocol for both. In the remaining children the median monitoring time with an $\mathrm{rStO}_{2}$ signal was 67.7 hours (range 0.9-71.4 hours) with 67.9 hours (interquartile range 64.7-69.0 hours) in the experimental group and 67.4 (interquartile range 63.9-68.9) hours in the control group. The variability in monitoring time was mainly due to death within the first three days of life (two infants were withdrawn from the trial by parental request and in four there were technical reasons for reduced NIRS recording time).

The baseline characteristics of the two groups were similar (table $1 \Downarrow)$. The 86 infants randomised to the experimental group had a median burden of hypoxia and hyperoxia of $36.1 \%$ hours (interquartile range 9.2-79.5\%hours) compared with 81.3 (38.5-181.3) \%hours in the 78 infants in the control group (fig $3 \Downarrow)$. In the experimental group this was a reduction of $58 \%$ ( $95 \%$ confidence interval $35 \%$ to $73 \%, \mathrm{P}<0.001$ ). The difference was primarily caused by a reduction in hypoxia $(\mathrm{P}=0.0012)$. 
None of the other secondary or exploratory outcomes differed statistically significantly between the groups (table $2 \Downarrow$ ). There was a trend towards better survival in the experimental group (table $3 \Downarrow$ ).

Of the recorded physiological variables and interventions during the intervention period in the first 72 hours of life, only the use of echocardiography and the occurrence and treatment of patent ductus arteriosus differed substantially (table $4 \Downarrow$ ).

The eight centres contributed 6, 8, 9, 11, 21, 36, 36, and 39 participants. The intervention effect showed significant heterogeneity among the centres $(\mathrm{P}<0.001)$. Removal of one centre with 36 infants resulted in the heterogeneity being insignificant $(\mathrm{P}=0.62)$. This centre had the largest reduction in burden in the intervention group. A post hoc sensitivity analysis reducing the mean effect of the intervention in this centre to a value equal to the mean effect in the rest of the centres resulted in a significant reduction in burden of $31 \%(\mathrm{P}=0.0497)$.

No severe adverse reactions were associated with the device, but 16 infants had skin marks from the NIRS sensors: four in the intervention group and 12 in the control group $(\mathrm{P}=0.03)$. The INVOS 5100C and NIRO 200NX were used in six and two centres, respectively.

\section{Discussion}

Cerebral oxygenation can be stabilised in extremely preterm infants during the first three days of life by using cerebral near infrared spectroscopy (NIRS) oximetry monitoring combined with a dedicated treatment guideline. The trial was not powered to detect differences in clinical outcomes, but we found an unexpected, borderline significant reduction of all cause mortality at term equivalent age in the NIRS group. We encountered no severe adverse reactions, but $10 \%$ of the infants developed skin marks possibly from pressure or heat from the NIRS sensors.

\section{Strengths and limitations of this study}

The strengths of the trial include early and continuous monitoring during the important transitional phase, utilisation of a specific and evidence based treatment guideline, ${ }^{18}$ dedicated software that supports the implementation of the treatment guideline, and a "burden alarm" that warns when hypoxia or hyperoxia is accumulating. Multiple centres and the use of several NIRS devices ensure greater generalisability if the findings. The limitations of cerebral NIRS are poor repeatability of measurements and differences between the varied devices, which we systematically dealt with by pretrial testing and comparisons between the devices. ${ }^{24} 25$

This study has several limitations. Firstly, repositioning of the NIRS sensor can change the regional tissue haemoglobin oxygen saturation $\left(\mathrm{rStO}_{2}\right) \cdot{ }^{12}{ }^{30}$ Out of range $\mathrm{rStO}_{2}$ values will on average tend to normalise after the sensor has been repositioned (regression towards the mean). This means that any repositioning motivated by out of range $\mathrm{rStO}_{2}$ values will introduce a bias on the primary outcome reducing out of range $\mathrm{rStO}_{2}$ only in the group with visible NIRS. Secondly, the combination of NIRS monitoring and a treatment guideline is a complex intervention and many factors influence how this interacts with the standard care of infants. The treatment guideline is a list of possible interventions and leaves the choice to the discretion of the attending clinical staff. One centre used cerebral NIRS as part of standard monitoring before the start of the trial; the remaining centres had no experience with $\mathrm{rStO}_{2}$ guided treatment. A learning curve would thus be expected. Thirdly, blinding of group allocation to clinical staff was not possible. This could lead to selective collateral intervention in one of the treatment groups leading to biased intervention effects. ${ }^{31-34} \mathrm{We}$ reduced the impact of this problem by centralised outcome assessments blinded to intervention group.

The intervention effect differed significantly among the centres. The centre causing this heterogeneity had four dedicated neonatologists doing all trial related clinical management, working in shifts during the intervention period of 72 hours, whereas the other centres had regular clinical staff attending the study participants. It is thus not surprising that this centre had the largest treatment effect. On the other hand it is also possible that bias was increased by close attention to the cerebral oximetry signal and repositioning of the sensor. We therefore did the sensitivity analysis, which demonstrated a reduced, but still significant effect. In future larger trials it will be important to ensure a more homogenous intervention by education of staff.

\section{Clinical outcomes}

The clinical outcomes did not differ significantly. In the experimental NIRS group we saw trends towards a reduction in severe brain injury and, surprisingly, all cause mortality. We assume that a reduction in burden of hypoxia and hyperoxia can reduce the risk of brain injury and impaired neurodevelopmental but not necessarily impact on survival. However, cerebral oxygenation may serve as an indicator of the sufficiency of systemic circulation and as such help to ensure sufficient oxygenation of other vital organs during the first critical days. The list of causes of death did not suggest any particular causal pathway. Another simple explanation for the potential benefit of being in the experimental group could be the extra attention paid to the infants by the clinical staff motivated by the alarms. In contrast, the trends towards increased risk of bronchopulmonary dysplasia and retinopathy of prematurity could represent unwanted effects of increased oxygenation. ${ }^{35}$ There was, however, no major differences in fractional inspired oxygen, peripheral capillary oxygen saturation or mean airway pressure (table 4). The record of interventions suggested a difference in approach only for echocardiography and detection and treatment of patent ductus arteriosus. The benefits of treating patent ductus arteriosus, however, remain contentious. ${ }^{36}$ Lastly, skin marks were more common in the control group. This may suggest poorer peripheral perfusion or may be simply due to less careful handling of the sensor in the blinded group.

\section{Trials of cerebral oximetry in other patient groups}

We have not been able to identify similar trials in this population. Two randomised clinical trials in adults during cardiac surgery compared visible cerebral NIRS monitoring with blinded NIRS monitoring. ${ }^{37} 38$ Murkin and colleagues reduced prolonged cerebral desaturations and found a shorter stay in the intensive care unit and less combined mortality and morbidity in the experimental group. ${ }^{37}$ Slater and colleagues found no difference in cerebral $\mathrm{rStO}_{2}$ between the two groups possibly due to poor implementation of the intervention. ${ }^{38}$ Both these trials had a predefined decrease from baseline $\mathrm{rStO}_{2}$ as a threshold for intervention, which is an approach to reduce the effect of the imprecision of NIRS oximetry. This is, however, not possible in neonatology as most ill infants are compromised from birth, thus the baseline $\mathrm{rStO}_{2}$ cannot be assumed to be safe. Furthermore, the sensor has to be repositioned repeatedly for long time monitoring. We have to rely on the absolute value of $\mathrm{rStO}_{2}$ and on absolute thresholds for intervention. 


\section{Trials in preterm infants with clinically relevant outcomes are needed}

The present results do not yet support the routine application of cerebral NIRS in extremely preterm infants. Although there were trends towards benefit and no record of serious adverse reactions, cerebral NIRS is demanding and may have unwanted effects. NIRS requires manipulation of vulnerable extremely preterm infants, requires additional staff time, and is costly in economical terms. As such, definitive evidence of benefit for improvement of clinically relevant outcomes is needed. We plan on doing a neurodevelopmental outcome assessment at two years corrected age; this phase II trial did not have the power to detect a difference. Therefore, further randomised clinical trials are needed. Such trials require a substantially greater number of infants to assess clinically relevant outcomes and thus require the involvement of many more centres. Several factors must be dealt with in the planning of such phase III trials. The appropriateness of the $\mathrm{rStO}_{2}$ thresholds for intervention should be reconsidered and new appraisals of the available NIRS oximeters should be made to take advantage of technical improvements. The treatment guideline should be reviewed. A training and certification programme for clinical staff should be provided before the start of such a trial.

\section{Conclusion}

In summary, the burden of cerebral hypoxia and hyperoxia was significantly reduced in extremely preterm infants monitored by NIRS and treated according to a dedicated treatment guideline. To assess the long term benefit and harm of the combination of NIRS and an updated treatment guideline, larger randomised clinical trials are needed.

We thank the families, nurses, doctors, and research assistants who took part in the trial; Jørn Wetterslev (Copenhagen Trial Unit) for help with the sample size estimation taking twins into consideration; and Heike Rabe (Southampton), Jan Miletin (Dublin), and Armin Kock (Tübingen) for their contribution as the data safety monitoring committee. The European Clinical Research Infrastructures Network (Ecrin.org) reviewed the protocol and supported the trial.

Local investigators: Paloma López-Ortego, María Carmen Bravo, Marta Ybarra, Fernando Cabañas, Berndt Urlesberger, Corinna Binder, Mmoloki Kenosi, Gavin Hawkes, Ida Sirgiovanni, Ita Herlihy, Andrea Edwards, and Cristine Sortica da Costa. Janus Engstrøm, data manager and electronic case record form developer, The Copenhagen Trial Unit, Centre for Clinical Intervention Research.

Contributors: SH-S, AP, TA, TAU, FvB, MB, OC, ED, MF, CG, BG, CH, $P L, A M P, P W, G P, W v O, G P, M W$ and $G G$ conceived and designed the study. SH-S, MB, CG, BG, CH, WvO, AMP, and GG designed the data collection instruments. JR designed the boxes for blinding the device displays and designed and maintained the NIRS data collection software. SH-S, AP, TA, TA, FvB, MB, OC, ED, ARF, MF, CH, PL, GP, AMP, LS, and GG assisted with acquisition of the data. SH-S, MB, CG, $\mathrm{CH}, \mathrm{WvO}, \mathrm{AMP}, \mathrm{PW}$, and $\mathrm{GG}$ analysed the data. SH-S, CG, and GG wrote the initial draft. SH-S, CG, BG, AMP, and GG provided administrative, technical, and material support and supervised the study. All authors critically reviewed revised versions of the paper and approved the final draft for submission. All the authors had access to the data and contributed substantially to the submitted report. SH-S and GG are the guarantors.

Funding: This work was supported by an unconditional and unrestricted grant from the Danish Council for Strategic Research (DKK 11,100,105). The funder had no role in the design, conduct, or analysis of the trial. Competing interests: All authors have completed the ICMJE uniform disclosure form at www.icmje.org/coi_disclosure.pdf and declare: no support from any organisation for the submitted work; no financial relationships with any organisations that might have an interest in the submitted work in the previous three years; no other relationships or activities that could appear to have influenced the submitted work.

Ethical approval: This study was approved by the local ethics committees: clinical research ethics committee of the Cork Teaching Hospitals (EMC 3 (xxx) 03/07/12); NRES Committee East of England-Cambridge South (12/EE/0329); Ethikkommission Medizinische Universität Graz (24-261 ex (11/12)); De Videnskabetiske Komiteer-Region Hovedstaden (H-4-2012-028); CPP Sud-Est III—Lyon (HCL/P 2012.728); Direzione Scientifica-Comitato di Etica-The IRCCS Fondazione Ca' Granda Ospedale Maggiore Policlinico (549/12-all.3); Medisch Ethische Toetsingscommissie-Universitair Medisch Centrum Utrecht (WAG/rc/12/ 036774); and Comité Ético de Investigación Clínica del Hospital Universitario "La Paz" de Madrid (15/2011). All participants gave informed consent before taking part in the trial.

Data sharing: Patient level data are available at from the corresponding author at request. Consent was not obtained but the presented data are anonymised and risk of identification is low.

Transparency: SH-S affirms that the manuscript is an honest, accurate, and transparent account of the study being reported; that no important aspects of the study have been omitted; and that any discrepancies from the study as planned (and, if relevant, registered) have been explained.

Hansen B, Hoff B, Greisen G, Mortensen EL for the Danish ETFOL study group. Early nasal continuous positive airway pressure in a cohort of the smallest infants in Denmark: neurodevelopmental outcome at five years of age. Acta Paediatrica 2004:93:190-5.

2 Serenius F, Sjörs G, Blennow M, Fellman V, Holmstrom G, Marsal K, et al. EXPRESS study shows significant regional differences in 1-year outcome of extremely preterm infants in Sweden. Acta Paediatr 2013;103:27-37.

3 Stoll BJ, Hansen NI, Bell EF, Shankaran S, Laptook AR, Walsh MC, et al. Neonatal outcomes of extremely preterm infants from the NICHD Neonatal Research Network. Pediatrics 2010;126:443-56.

4 Noori S, Stavroudis TA, Seri I. Systemic and cerebral hemodynamics during the transitional period after premature birth. Clin Perinatol 2009;36:723-36-v.

5 Hunt RW, Evans N, Rieger I, Kluckow M. Low superior vena cava flow and neurodevelopment at 3 years in very preterm infants. J Pediatr 2004;145:588-92.

6 Kluckow M, Evans N. Superior vena cava flow in newborn infants: a novel marker of systemic blood flow. Arch Dis Child Fetal Neonatal Ed 2000;82:F182-7.

7 Wolf M, Greisen G. Advances in near-infrared spectroscopy to study the brain of the preterm and term neonate. Clin Perinatol 2009;36:807-34, vi.

8 Greisen G, Leung T, Wolf M. Has the time come to use near-infrared spectroscopy as a routine clinical tool in preterm infants undergoing intensive care? Phil Trans $R$ Soc $A$ 2011;369:4440-51.

9 Wintermark P, Hansen A, Warfield SK, Dukhovny D, Soul JS. Near-infrared spectroscopy versus magnetic resonance imaging to study brain perfusion in newborns with hypoxic-ischemic encephalopathy treated with hypothermia. Neuroimage 2014;85(Pt 1)287-93.

10 Taussky P, O'Neal B, Daugherty WP, Luke S, Thorpe D, Pooley RA, et al. Validation of frontal near-infrared spectroscopy as noninvasive bedside monitoring for regional cerebral blood flow in brain-injured patients. Neurosurg Focus 2012;32:E2.

11 Alderliesten T, Lemmers PMA, van Haastert IC, de Vries LS, Bonestroo HJC, Baerts W, et al. Hypotension in preterm neonates: low blood pressure alone does not affect neurodevelopmental outcome. J Pediatr 2014;164:986-91.

12 Sorensen LC, Greisen G. Precision of measurement of cerebral tissue oxygenation index using near-infrared spectroscopy in preterm neonates. J Biomed Opt 2006;11:054005.

13 Noori S, McCoy M, Anderson MP, et al. Changes in cardiac function and cerebral blood flow in relation to peri/intraventricular hemorrhage in extremely preterm infants. $J$ Pediatr 2014;164:264-70.e1-3.

14 Kurth CD, Levy WJ, McCann J. Near-infrared spectroscopy cerebral oxygen saturation thresholds for hypoxia-ischemia in piglets. J Cereb Blood Flow Metab 2002;22:335-41.

15 Kurth CD, Mccann JC, Wu J, Miles L, Loepke AW. Cerebral oxygen saturation-time threshold for hypoxic-ischemic injury in piglets. Anesth Analg 2009;108:1268-77.

16 Gerstner B, DeSilva TM, Genz K, Armstrong A, Brehmer F, Neve RL, et al. Hyperoxia causes maturation-dependent cell death in the developing white matter. $J$ Neurosci 2008;28:1236-45.

17 Yis U, Kurul SH, Kumral A, Cilaker S, Tugyan K, Genc S, et al. Hyperoxic exposure leads to cell death in the developing brain. Brain Dev 2008;30:556-62.

18 Pellicer A, Greisen G, Benders M, Claris O, Dempsey EM, Fumagalli M, et al. The SafeBoosC phase ii randomised clinical trial: a treatment guideline for targeted near-infrared-derived cerebral tissue oxygenation versus standard treatment in extremely preterm infants. Neonatology 2013;104:171-8

19 Hyttel-Sørensen S, Austin T, van Bel F, Benders M, Claris O, Dempsey EM, et al. Clinical use of cerebral oximetry in extremely preterm infants is feasible. Dan Med J 2013;60:A4533.

20 Hyttel-Sørensen S, Austin T, van Bel F, Benders M, Claris O, Dempsey EM, et al. A phase II randomized clinical trial on cerebral near-infrared spectroscopy plus a treatment guideline versus treatment as usual for extremely preterm infants during the first three days of life (SafeBoosC): study protocol for a randomized controlled trial. Trials 2013;14:120.

21 E6 (R1) Guideline for Good Clinical Practice. International Conference on Harmonisation 2009;1-48. www.ema.europa.eu/docs/en_GB/document library/Scientific quideline/2009/ 09/WC500002874.pdf. 


\section{What is already known on this topic}

Extremely preterm infants are at risk of neurodevelopmental impairment

Cerebral near infrared spectometry (NIRS) oximetry enables continuous estimation of the oxygen balance of the brain tissue and has been shown to correlate with clinical outcome

The possibility of cerebral oxygen imbalance being reduced by a dedicated treatment guideline in extremely preterm infants has not yet been tested in a randomised trial

\section{What this study adds}

The combination of cerebral NIRS oximetry and a dedicated treatment guideline reduced the amount of oxygen imbalance in the brain of extremely preterm infants during the first three days of life

No severe adverse reactions were associated with the device

Larger trials powered for relevant clinical outcomes are needed to test the clinical relevance of the present findings

22 Dix LM, van Bel F, Baerts W, Lemmers PM. Comparing near-infrared spectroscopy devices and their sensors for monitoring regional cerebral oxygen saturation in the neonate. Pediatr Res 2013;74:557-63.

23 Dullenkopf A, Frey B, Baenziger O, Gerber A, Weiss M. Measurement of cerebral oxygenation state in anaesthetized children using the INVOS 5100 cerebral oximeter Pediatr Anesth 2003;13:384-91.

24 Hyttel-Sørensen S, Kleiser S, Wolf M, Greisen G. Calibration of a prototype NIRS oximeter against two commercial devices on a blood-lipid phantom. Biomed Opt Express 2013;4:1662.

25 Hyttel-Sørensen S, Sorensen LC, Riera J, Greisen G. Tissue oximetry: a comparison of mean values of regional tissue saturation, reproducibility and dynamic range of four NIRS-instruments on the human forearm. Biomed Opt Express 2011;2:3047-57.

26 Hyttel-Sørensen S, Hessel TW, Greisen G. Peripheral tissue oximetry: comparing three commercial near-infrared spectroscopy oximeters on the forearm. $J$ Clin Monit Comput 2014:28:149-55

27 Walsh MC, Kliegman RM. Necrotizing enterocolitis: treatment based on staging criteria. Pediatr Clin North Am 1986;33:179-201.

28 International Committee for the Classification of Retinopathy of Prematurity. The International Classification of Retinopathy of Prematurity revisited. 2005; 991-9.

29 Rao JN, Scott AJ. A simple method for the analysis of clustered binary data. Biometrics 1992:48:577-85.

30 Hessel TW, Hyttel-Sørensen S, Greisen G. Cerebral oxygenation after birth-a comparison

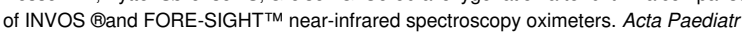
2014;103:488-93.

31 Savović J, Jones HE, Altman DG, Harris RJ, Juni P, Pildal J, et al. Influence of reported study design characteristics on intervention effect estimates from randomized, controlled trials. Ann Intern Med 2012;157:429-38.

32 Kjaergard LL, Villumsen J, Gluud C. Reported methodologic quality and discrepancie between large and small randomized trials in meta-analyses. Ann Intern Med 2001;135:982-9.
33 Wood L, Egger M, Gluud LL, Schulz KF, Juni P, Altman DG, et al. Empirical evidence of bias in treatment effect estimates in controlled trials with different interventions and outcomes: meta-epidemiological study. BMJ 2008;336:601-5.

34 Schulz KF, Chalmers I, Hayes RJ, Altman DG. Empirical evidence of bias: dimensions of methodological quality associated with estimates of treatment effects in controlled trials. JAMA 1995;273:408-12.

35 SUPPORT Study Group of the Eunice Kennedy Shriver NICHD Neonatal Research Network, Finer NN, Carlo WA, et al. Early CPAP versus surfactant in extremely preterm infants. N Engl J Med 2010;362:1970-9.

36 Benitz WE. Treatment of persistent patent ductus arteriosus in preterm infants: time to accept the null hypothesis? J Perinatol 2010;30:241-52.

37 Murkin JM, Adams SJ, Novick RJ, Quantz M, Bainbridge D, Iglesias I, et al. Monitoring brain oxygen saturation during coronary bypass surgery: a randomized, prospective study. Anesth Analg 2007:104:51-8.

38 Slater JP, Guarino T, Stack J, Vinod K, Bustami RT, Brown JM, et al.Cerebral oxygen desaturation predicts cognitive decline and longer hospital stay after cardiac surgery. Ann Thorac Surg 2009;87:36-44; discussion 44-5.

\section{Accepted: 21 November 2014}

\section{Cite this as: BMJ 2015:350:97635}

This is an Open Access article distributed in accordance with the Creative Commons Attribution Non Commercial (CC BY-NC 4.0) license, which permits others to distribute, remix, adapt, build upon this work non-commercially, and license their derivative works on different terms, provided the original work is properly cited and the use is non-commercial. See: http://creativecommons.org/licenses/by-nc/4.0/. 


\section{Tables}

Table 1| Baseline characteristics of infants. Values are numbers (percentages) unless stated otherwise

\begin{tabular}{lcc} 
Characteristics & NIRS ( $\mathbf{n}=\mathbf{8 6})$ & Blinded NIRS (control) $(\mathbf{n}=\mathbf{8 0})$ \\
Median (range) birth weight (g) & $806(410-1286)$ & $880(490-1330)$ \\
\hline Median (interquartile range) gestational age at birth (weeks) & $26.6(25.7-27.4)$ & $26.8(25.5-27.6))$ \\
\hline Gestational age <26 weeks & $28(33)$ & $25(31)$ \\
\hline Male sex & $44(51)$ & $34(43)$ \\
\hline Twins & $20(23)$ & $14(18)$ \\
\hline Prenatal steroids (complete course) & $58(67)$ & $76(71)$ \\
\hline Prolonged rupture of membranes & $26(31)$ & $72(40)$ \\
\hline Maternal clinical chorioamnionitis & $6(7)$ & $7.31(0.096)$ \\
\hline Apgar score $\leq 5$ at 5 minutes & $15(18)$ & \\
\hline Mean (SD) umbilical arterial pH & $7.33(0.088)$ & \\
\hline NIRS=near infrared spectroscopy oximetry. & & \\
\hline
\end{tabular}




\begin{tabular}{|c|c|c|c|c|c|}
\hline Outcomes & NIRS ( $n=86)$ & $\begin{array}{l}\text { Blinded NIRS (control) } \\
\qquad(n=80)\end{array}$ & $\begin{array}{l}\text { Relative change in \% } \\
\qquad(95 \% \mathrm{Cl})\end{array}$ & $\begin{array}{l}\text { Adjusted relative risk } \\
\qquad(95 \% \mathrm{Cl})\end{array}$ & $\begin{array}{l}\text { Adjusted P value } \\
\text { (unadjusted) }\end{array}$ \\
\hline \multicolumn{6}{|l|}{ Primary } \\
\hline $\begin{array}{l}\text { Median (interquartile range) burden } \\
\text { of hypoxia and hyperoxia (\%hours) }\end{array}$ & $36.1(9.2-79.5)$ & $81.3(38.5-181.3)(n=78)$ & $-58(-35$ to -74$)$ & NA & $<0.001^{*}$ \\
\hline \multicolumn{6}{|l|}{ Secondary } \\
\hline All cause mortality at term & $12 / 86(14)$ & $20 / 80(25)$ & NA & $0.50(0.29$ to 1.00$)$ & $0.10(0.049)$ \\
\hline \multicolumn{6}{|l|}{$\begin{array}{l}\text { Brain injury on cerebral } \\
\text { ultrasonography: }\end{array}$} \\
\hline None & $21 / 80(26)$ & 26/77 (34) & NA & - & $0.11(0.053)$ \\
\hline Mild-moderate & $49 / 80(61)$ & $33 / 77(43)$ & NA & - & \\
\hline Severe & $10 / 80(13)$ & $18 / 77(23)$ & NA & - & \\
\hline \multicolumn{6}{|l|}{ Exploratory } \\
\hline $\begin{array}{l}\text { Median (interquartile range) burden } \\
\text { of hypoxia (\%hours) }\end{array}$ & $16.6(5.4-68.1)$ & $53.6(17.4-171.3)(n=78)$ & $-58(-24$ to -76$)$ & NA & $0.0012^{*}$ \\
\hline $\begin{array}{l}\text { Mean (SD) burden of hyperoxia } \\
\text { (\%hours) }\end{array}$ & $1.2(0.3-9.6)$ & $1.1(0.1-23.4)(n=78)$ & $1(-35$ to 194$)$ & NA & $0.98^{\star}$ \\
\hline Necrotising enterocolitis $\dagger$ & $9 / 86(11)$ & $10 / 80(13)$ & NA & $0.83(0.33$ to 1.94$)$ & 0.69 \\
\hline Bronchopulmonary dysplasiał & $41 / 72(57)$ & $28 / 60(47)$ & NA & $1.27(0.94$ to 1.50$)$ & 0.20 \\
\hline Retinopathy of prematurity§ & $14 / 86(16)$ & $8 / 80(10)$ & NA & $1.64(0.75$ to 3.00$)$ & 0.20 \\
\hline \multicolumn{6}{|l|}{ NA=not applicable. } \\
\hline \multicolumn{6}{|c|}{$\begin{array}{l}\text { *Data were log transformed to normalise distributions. } P \text { values originate from analysis with adjustment for gestational age category and trial site indicator and are } \\
\text { adjusted for multiplicity. Medians and interquartile ranges are based on raw data obtained before transformation. } \\
\text { †Modified Bell's stage } 2 \text { or } 3 .\end{array}$} \\
\hline
\end{tabular}


Table 3| Causes of death

\begin{tabular}{lcccccc} 
Group & GMH-IVH/PVL & RDS/respiratory failure & Circulatory failure & $\begin{array}{c}\text { Necrotising } \\
\text { enterocolitis }\end{array}$ & Infection/sepsis & Other \\
NIRS & 1 & 4 & 3 & 1 & 0 & 3 \\
\hline Blinded NIRS (control) & 2 & 6 & 3 & 3 & 2 \\
\hline
\end{tabular}

GMH-IVH/PVL=germinal matrix-intraventricular haemorrhage /periventricular leukomalacia; RDS=respiratory distress syndrome; NIRS=near infrared spectroscopy oximetry. 
Table 4| Death, interventions, and physiological variables during intervention period of first 72 hours of life. Values are numbers (percentages) unless stated otherwise

\begin{tabular}{|c|c|c|}
\hline Variables & NIRS $(n=86)$ & Blinded NIRS (control) $(n=80)$ \\
\hline Deaths & $3(3.5)$ & $6(7.5)$ \\
\hline \multicolumn{3}{|l|}{ Respiratory: } \\
\hline Surfactant administered & $64(74.4)$ & $63(78.8)$ \\
\hline Mechanical ventilation & $57(66.3)$ & $54(67.5)$ \\
\hline Mean (SD) postnatal age at onset (hours) & $3.5(9.8)$ & $3.4(8.7)$ \\
\hline Mean (SD) duration (hours) & $52.2(24.9)$ & $53.0(25.2)$ \\
\hline \multicolumn{3}{|l|}{ Circulatory: } \\
\hline Echocardiography performed & $53(61.6)$ & $38(47.5)$ \\
\hline Haemodynamic significant patent ductus arteriosus & $19(22.1)$ & $9(11.3)$ \\
\hline Patent ductus arteriosus treated & $16(18.6)$ & $5(6.3)$ \\
\hline Use of vasopressors/inotropes & $20(23.3)$ & $19(23.8)$ \\
\hline Mean (SD) fluid boluses & $0.6(1.2)$ & $0.9(1.9)$ \\
\hline Any red blood cell transfusion & $24(27.9)$ & $27(33.8)$ \\
\hline Corticosteroids & $2(2.3)$ & $6(7.5)$ \\
\hline Mean (SD) No of blood samples & $11.1(8.0)$ & $11.5(8.9)$ \\
\hline \multicolumn{3}{|l|}{ Physiological variables*: } \\
\hline Mean (SD) fractional inspired oxygen (\%) & $26.6(4.2)$ & $25.4(3.8)$ \\
\hline Mean (SD) peripheral capillary oxygen saturation (\%) & $93.6(12.5)$ & $93.6(9.1)$ \\
\hline Mean (SD) mean airway pressure $\left(\mathrm{cm} \mathrm{H}_{2} \mathrm{O}\right)$ & $7.9(4.1)$ & $7.5(3.3)$ \\
\hline Mean (SD) heart rate (beats/min) & $155.4(14.6)$ & $152.8(14.3)$ \\
\hline Mean (SD) mean arterial blood pressure $(\mathrm{mm} \mathrm{Hg})$ & $35.9(8.4)$ & $35.6(7.1)$ \\
\hline
\end{tabular}

NIRS=near infrared spectroscopy oximetry.

${ }^{*}$ Recorded six hourly. Data were entered at1348 time points. Missing data were due to technical issues or death of child before 72 hours of life. 


\section{Figures}

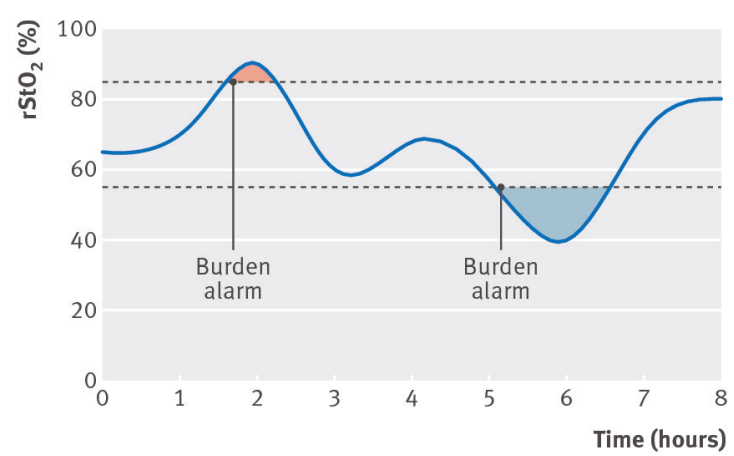

Fig 1 Schematic illustration of concept of burden of hypoxia and hyperoxia and the alarm. $\mathrm{rStO}_{2}=$ regional tissue haemoglobin oxygen saturation

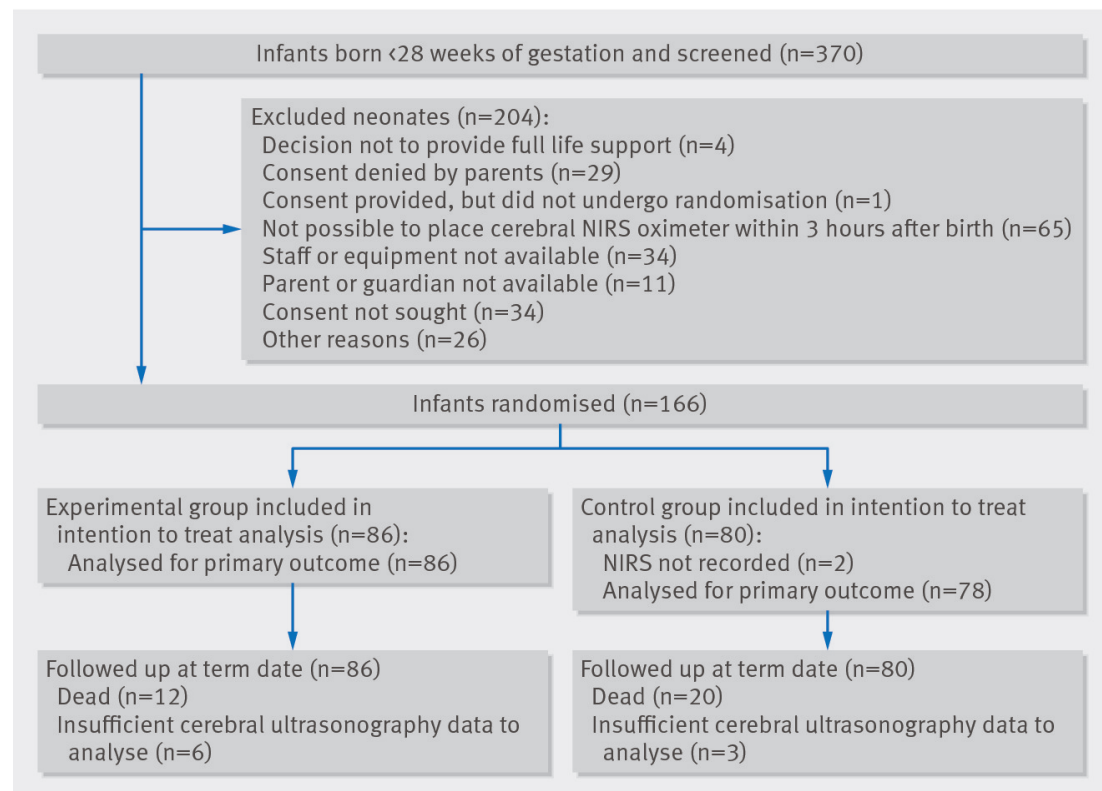

Fig 2 Flow of participants through study. NIRS=cerebral near infrared spectroscopy oximetry

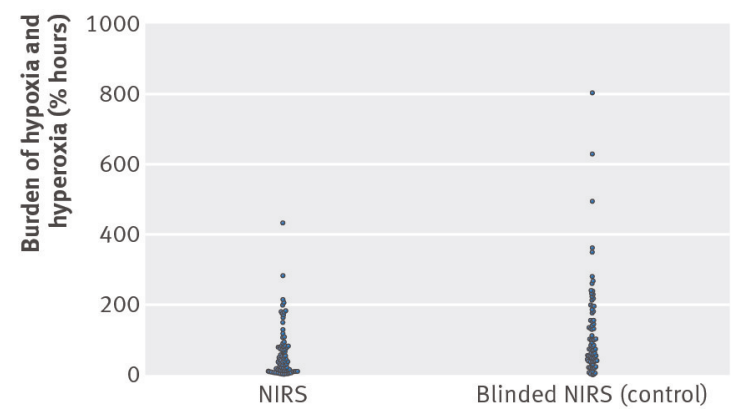

Fig 3 Burden of hypoxia and hyperoxia by treatment group. NIRS=cerebral near infrared spectroscopy oximetry 